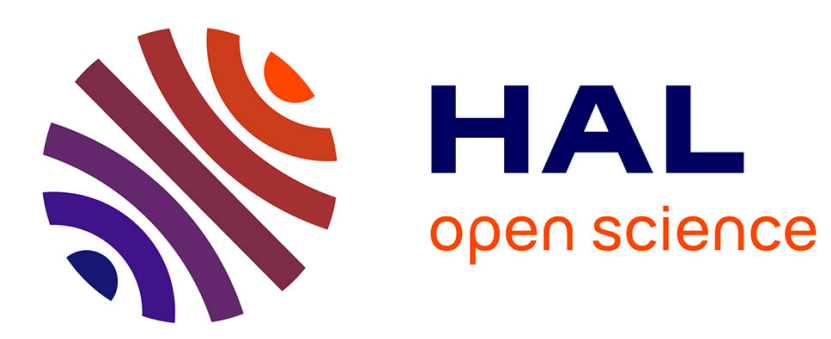

\title{
New statistical measures of symmetry breaking in the ground state region of nuclei
}

\author{
C. Quesne, S. Spitz
}

\section{To cite this version:}

C. Quesne, S. Spitz. New statistical measures of symmetry breaking in the ground state region of nuclei. Journal de Physique Lettres, 1977, 38 (13), pp.237-240. 10.1051/jphyslet:019770038013023700 . jpa-00231367

\section{HAL Id: jpa-00231367 https://hal.science/jpa-00231367}

Submitted on 1 Jan 1977

HAL is a multi-disciplinary open access archive for the deposit and dissemination of scientific research documents, whether they are published or not. The documents may come from teaching and research institutions in France or abroad, or from public or private research centers.
L'archive ouverte pluridisciplinaire HAL, est destinée au dépôt et à la diffusion de documents scientifiques de niveau recherche, publiés ou non, émanant des établissements d'enseignement et de recherche français ou étrangers, des laboratoires publics ou privés. 


\title{
NEW STATISTICAL MEASURES OF SYMMETRY BREAKING IN THE GROUND STATE REGION OF NUCLEI
}

\author{
C. QUESNE $\left({ }^{*}\right)$ and S. SPITZ $\left({ }^{* *}\right)$ \\ Physique Théorique et Mathématique, Université Libre de Bruxelles, Bruxelles, Belgium
}

(Reçu le 5 mai 1977, accepté le 6 juin 1977)

\begin{abstract}
Résumé. - Nous définissons deux mesures statistiques de violation de symétrie qui tiennent compte de tous les moments internes des sous-espaces irréductibles et peuvent être utilisées dans la région de l'état fondamental des noyaux. Nous testons leur validité dans un modèle exactement soluble.
\end{abstract}

Abstract. - We define two statistical measures of symmetry breaking which take all the internal moments of the irreducible subspaces into account, and can be used in the ground state region of nuclei. We test their validity in an exactly solvable model.

During the last few years, French's spectral distribution method [1-3] has been extensively used to study the goodness of various group symmetries in nuclei [3-8]. The usefulness of the method stems from the fact that many results can be deduced from the first two moments - centroid energies and total variances (squares of the widths) - of the Hamiltonian averaged over the states in an irreducible representation of the group, and from the decomposition of the total widths into partial widths, which connect a given irreducible subspace with itself (internal width) or with the others (mixing widths). From the centroid energies and partial widths, statistical measures of symmetry breaking can indeed be constructed [9-11]. In this paper, we introduce two new parameters which can be used to give a measure of the amount of admixture in the ground state region of nuclei. We study also their validity by comparison with exact shell model results obtained in a solvable model, and show that in many cases they are a great improvement on another commonly used measure of symmetry breaking.

Let us denote by $\mathrm{S}(\lambda), \lambda=1,2, \ldots, r$, the $r$ irreducible subspaces of dimension $d(\lambda)$ of the spectroscopic space $S$ and assume that the vectors $|\lambda i\rangle, i=1,2, \ldots, d(\lambda)$, form a basis of $\mathrm{S}(\lambda)$. For a given Hamiltonian $H$, the centroid energy, total and partial variances of the distribution of the states belonging to $S(\lambda)$ are defined as

(*) Maître de recherches F.N.R.S.

$\left.{ }^{* *}\right)$ Chercheur agréé, I.I.S.N.

$$
\begin{aligned}
E(\lambda) & =[d(\lambda)]^{-1} \sum_{i=1}^{d(\lambda)}\langle\lambda i|H| \lambda i\rangle \\
\sigma^{2}(\lambda) & =[d(\lambda)]^{-1} \sum_{i=1}^{d(\lambda)}\left\langle\lambda i\left|H^{2}\right| \lambda i\right\rangle-[E(\lambda)]^{2}
\end{aligned}
$$

and

$$
\begin{aligned}
\sigma^{2}\left(\lambda \rightarrow \lambda^{\prime}\right)= & {[d(\lambda)]^{-1} \sum_{i=1}^{d(\lambda)} \sum_{j=1}^{d\left(\lambda^{\prime}\right)} x } \\
& \times\left|\left\langle\lambda^{\prime} j|H| \lambda i\right\rangle\right|^{2}-\delta_{\lambda \lambda}[E(\lambda)]^{2}
\end{aligned}
$$

respectively. The $r$ irreducible subspaces are assumed to be arranged in order of increasing centroid energy, i.e., $E(1)<E(2)<\cdots<E(r)$.

The exact eigenstates of $H$ in the spectroscopic space $\mathrm{S}$, corresponding to the eigenvalues $E_{\alpha}$, can be written as

$$
\left|\Psi_{\alpha}\right\rangle=\left\{\sum_{\lambda^{\prime}=1}^{r} \sum_{j=1}^{d\left(\lambda^{\prime}\right)}\left[a_{\lambda^{\prime} j}^{(\alpha)}\right]^{2}\right\}^{-1 / 2} \sum_{\lambda=1}^{r} \sum_{i=1}^{d(\lambda)} a_{\lambda i}^{(\alpha)}|\lambda i\rangle,
$$

where $\alpha=1,2, \ldots, \sum_{\lambda=1}^{r} d(\lambda)$, and $a_{\lambda i}^{(\alpha)}$ are some real, unknown constants. Among these states, we choose the $d(1)$ states which have the biggest components in the subspace $S(1)$. According to the centroid spectrum, they should include the lowest few eigenstates of $H$. We designate them by $\left|\Psi_{i}\right\rangle, i=1,2, \ldots, d(1)$, and their corresponding eigenvalues by $E_{i}$. The mixing ratios

$$
M_{i}^{2}(1 \rightarrow \lambda) \equiv\left\{\sum_{j=1}^{d(1)}\left[a_{1 j}^{(i)}\right]^{2}\right\}^{-1} \sum_{k=1}^{d(\lambda)}\left[a_{\lambda k}^{(i)}\right]^{2}, \quad \lambda=2, \ldots, r,
$$


measure the relative importance of the components belonging to the subspaces $S(\lambda)$ and $S(1)$ in the eigenstate $\left|\Psi_{i}\right\rangle$. In terms of these, the total intensities of the components of $\left|\Psi_{i}\right\rangle$ in the subspaces $S(1)$ and $\mathrm{S}(\lambda), \lambda=2, \ldots, r$ are given by

$$
\left\{1+\sum_{\lambda^{\prime}=2}^{r} M_{i}^{2}\left(1 \rightarrow \lambda^{\prime}\right)\right\}^{-1}
$$

and

$$
\begin{array}{r}
M_{i}^{2}(1 \rightarrow \lambda) \times\left\{1+\sum_{\lambda^{\prime}=2}^{r} M_{i}^{2}\left(1 \rightarrow \lambda^{\prime}\right)\right\}^{-1}, \\
\lambda=2, \ldots, r,
\end{array}
$$

respectively.

It is generally admitted in the spectral distribution method $[9,10]$ that the average of $M_{i}^{2}(1 \rightarrow \lambda)$ over the states $\left|\Psi_{i}\right\rangle, i=1, \ldots, d(1)$, can be approximated by the (perturbative) parameter

$$
I^{2}(1 \rightarrow \lambda) \equiv \sigma^{2}(1 \rightarrow \lambda) \times[E(1)-E(\lambda)]^{-2},
$$

whenever the latter is very small in comparison with unity, i.e., the mixing widths $\sigma(1 \rightarrow \lambda)$ are very small and the centroids of the subspaces $S(1)$ and $S(\lambda)$ are well separated. In this case, the total intensities of the components of an average state of the set

$$
\left.\left\{\left|\Psi_{i}\right\rangle\right\} i=1, \ldots, d(1)\right\}
$$

in the subspaces $\mathrm{S}(1)$ and $\mathrm{S}(\lambda), \lambda=2, \ldots, r$, are approximated by

$$
\left\{1+\sum_{\lambda^{\prime}=2}^{r} I^{2}\left(1 \rightarrow \lambda^{\prime}\right)\right\}^{-1}
$$

and

$I^{2}(1 \rightarrow \lambda) \times\left\{1+\sum_{\lambda^{\prime}=2}^{r} I^{2}\left(1 \rightarrow \lambda^{\prime}\right)\right\}^{-1}, \lambda=2, \ldots, r$,

respectively. In practice however, the $I^{2}$ parameter is extensively used as a qualitative measure of symmetry breaking even when it is not very small [4-10].

As was already pointed out in references [6] and [7], the $I^{2}$ parameter only uses part of the information we have at our disposal regarding the moments of the distributions. The dimensions of the subspaces enter the definition of $I^{2}$ in an indirect way through the definitions (1) and (3) of the centroid energies and partial widths. On the other hand, the internal widths $\sigma(\lambda \rightarrow \lambda)$ of the subspaces $\mathrm{S}(\lambda), \lambda=1, \ldots, r$, which are mostly due to the non-degeneracy of the states belonging to $S(\lambda)$, are not taken at all into account. This is not important if they are small compared with the distances between the centroids. In many cases however they may be rather large $[6,7]$. If moreover the mixing widths are small, the amount of admixture of the subspaces $S(\lambda), \lambda=2, \ldots, r$, into $S(1)$ varies quite a lot when going through the subset

$$
\left.\left\{\left|\Psi_{i}\right\rangle\right\} i=1, \ldots, d(1)\right\} .
$$

As the $I^{2}$ parameter gives information about an average eigenstate which has then nothing to do with the lowest lying states we are interested in, it becomes rather useless. The purpose of this paper is to present two new measures of symmetry breaking which are intended to give detailed information about the admixtures in the low lying states even when the $I^{2}$ parameter fails. We now proceed to derive them.

The Hamiltonian matrix $\mathscr{H}$ in the spectroscopic space $S$ can be partitioned according to the irreducible subspaces $\mathrm{S}(\lambda)$. Let $\mathcal{H}_{\lambda \lambda^{\prime}}, \lambda, \lambda^{\prime}=1, \ldots, r$, be the $d(\lambda) \times d\left(\lambda^{\prime}\right)$ submatrix corresponding to the subspaces $S(\lambda)$ and $S\left(\lambda^{\prime}\right)$. The first step of the derivation consists in replacing the matrix $\mathscr{T}$ by a statistical ensemble $\{\mathscr{K}\}$ of real, symmetrical matrices which have the same dimension as $\mathscr{H}$ and reproduce as many partial moments of $\mathcal{H}$ as possible. The ensemble $\{\pi\}$ we consider in the following [6] reproduces all the internal moments of $\mathcal{H}$ (exactly or approximately depending on whether the dimensions $d(\lambda)$ are small or large). Moreover "it reproduces the mixing widths $\sigma\left(\lambda \rightarrow \lambda^{\prime}\right), \lambda \neq \lambda^{\prime}$, of $\mathscr{H}$. The first condition can be reformulated in the following way : (C1) The diagonal blocks $\varkappa_{\lambda \lambda}$ are identified with the corresponding blocks $H_{\lambda \lambda}$, exactly or approximately diagonalized; the diagonal elements of $\varkappa_{\lambda \lambda}$ are therefore the exact or approximate eigenvalues $E_{i}(\lambda), i=1, \ldots, d(\lambda)$, of $\mathcal{H e}_{\lambda \lambda}$. The procedure we use to get the approximate $E_{i}(\lambda)$, whenever $d(\lambda)$ is large, is the well known Ratcliff's method [2]: It should be stressed that this procedure is entirely based on a calculation of moments, and therefore does not imply any explicit construction of the matrix elements of $\mathscr{H}_{\lambda \lambda}$. The second condition can be satisfied in many ways. We choose to do it as follows : (C2) The matrix elements of each of the off-diagonal blocks $\aleph_{\lambda \lambda^{\prime}}\left(\lambda \neq \lambda^{\prime}\right)$ have the same magnitude equal to $\sigma\left(\lambda \rightarrow \lambda^{\prime}\right) / \sqrt{d\left(\lambda^{\prime}\right)}$, but random signs.

The second step of the derivation consists in dividing the matrices $\kappa$ into two matrices, $K=\varkappa_{0}+K_{1}$, where $\varkappa_{0}$ contains only the diagonal elements and $\mathcal{K}_{1}$ only the off-diagonal elements. First order perturbation theory is then used with $\varkappa_{0}$ as the unperturbed Hamiltonian matrix and $K_{1}$ as the perturbation. The eigenstates $|1 i\rangle, i=1, \ldots, d(1)$, of $\varkappa_{0}$ belonging to $S(1)$ are changed into

$$
\begin{aligned}
\left|\Phi_{i}\right\rangle= & \left\{1+\sum_{\lambda^{\prime}=2}^{r}\left[d\left(\lambda^{\prime}\right)\right]^{-1} \sigma^{2}\left(1 \rightarrow \lambda^{\prime}\right) \sum_{k=1}^{d\left(\lambda^{\prime}\right)}\left[E_{i}(1)-E_{k}\left(\lambda^{\prime}\right)\right]^{-2}\right\}^{-1 / 2} \times \\
& \times\left\{|1 i\rangle+\sum_{\lambda=2}^{r}[d(\lambda)]^{-1 / 2} \sigma(1 \rightarrow \lambda) \sum_{j=1}^{d(\lambda)} \varepsilon_{1 i, \lambda j}\left[E_{i}(1)-E_{j}(\lambda)\right]^{-1}|\lambda j\rangle\right\}, \quad i=1, \ldots, d(1),
\end{aligned}
$$


where $\varepsilon_{1 i, \lambda j}$ is the random sign of the matrix element $\mathcal{K}_{1 i, \lambda j}$. It is then straightforward to see that the total intensities of the components of $\left|\Phi_{i}\right\rangle$ in the subspaces $\mathrm{S}(1)$ and $\mathrm{S}(\lambda), \lambda=2, \ldots ; r$, are given by expressions similar to those contained in eq. (6) and (7) respectively, if we replace there $M_{i}^{2}(1 \rightarrow \lambda)$ by [6]

$J_{i}^{2}(1 \rightarrow \lambda) \equiv[d(\lambda)]^{-1} \sigma^{2}(1 \rightarrow \lambda) \sum_{j=1}^{d(\lambda)}\left[E_{i}(1)-E_{j}(\lambda)\right]^{-2}$

This is true for any matrix of the ensemble, which means the ensemble deviation is zero. The parameters $J_{i}^{2}(1 \rightarrow \lambda)$, where $i=1, \ldots, d(1)$, and $\lambda=2, \ldots, r$, measure the amount of admixture in the states of $S(1)$.

Had we chosen the ensemble $\{\pi\}$ so as to reproduce only the centroid energies of the subspaces $S(\lambda)$, in addition to the mixing widths $\sigma\left(\lambda \rightarrow \lambda^{\prime}\right), \lambda \neq \lambda^{\prime}$, by writing $\mathcal{K}_{\lambda \lambda}=E(\lambda)$. 1, where 1 is the unit matrix of dimension $d(\lambda)$, we should have obtained the $I^{2}(1 \rightarrow \lambda)$ parameter defined in eq. (8) instead of $J_{i}^{2}(1 \rightarrow \lambda)[9,10]$. We see therefore that the only difference between these two parameters is that the latter contains additional information about the internal widths $\sigma(\lambda \rightarrow \lambda)$ as well as all the higher internal moments. It is clear from eq. (8) and (12) that when the internal widths of $S(1)$ and $S(\lambda)$ become negligible compared with the distance between their centroids, each $J_{i}^{2}(1 \rightarrow \lambda)$ goes to $I^{2}(1 \rightarrow \lambda)$, as should be the case. When the internal widths are not small, $J_{i}^{2}(1 \rightarrow \lambda)$ varies from one state to another, and thus we may hope to be able to use the $J_{i}^{2}(1 \rightarrow \lambda)$ to determine whether certain low-lying states of $S(1)$ remain approximately pure.

When the eigenvalue $E_{i}(1)$ is close to one of the eigenvalues $E_{j}(2), J_{i}^{2}(1 \rightarrow \lambda)$ becomes very large. One must then try to go beyond ordinary first order perturbation theory. When $E_{i}(1)-E_{j}(\lambda)$ is not too small, a great improvement may be achieved by replacing the first order Rayleigh-Schrödinger (R-S) approximation by the first order Brillouin-Wigner (B-W) approximation [12]. Using the same ensemble of matrices $\{\mathcal{K}\}$ as above, it is straightforward to show that in the expression (11) of $\left|\Phi_{i}\right\rangle, E_{i}(1)$ is merely replaced by the first order B-W approximation $\bar{E}_{i}$ for the energy of the $i$ th state of $\mathrm{S}(1)$, so that $J_{i}^{2}(1 \rightarrow \lambda)$ is changed into

$K_{i}^{2}(1 \rightarrow \lambda) \equiv[d(\lambda)]^{-1} \sigma^{2}(1 \rightarrow \lambda) \sum_{j=1}^{d(\lambda)}\left[\bar{E}_{i}-E_{j}(\lambda)\right]^{-2}$.

The energy $\bar{E}_{i}$ is a solution of the following equation :

$$
\begin{aligned}
\bar{E}_{i}=E_{i}(1)+\sum_{\lambda^{\prime}=2}^{r}\left[d\left(\lambda^{\prime}\right)\right]^{-1} \sigma^{2}\left(1 \rightarrow \lambda^{\prime}\right) \times \\
\times \sum_{k=1}^{d\left(\lambda^{\prime}\right)}\left[\bar{E}_{i}-E_{k}\left(\lambda^{\prime}\right)\right]^{-1} .
\end{aligned}
$$

In fact this equation has $\left[1+\sum_{\lambda^{\prime}=2}^{r} d\left(\lambda^{\prime}\right)\right]$ solutions. If the perturbation calculation is to be meaningful, we have to choose among them the solution which is closest to $E_{i}(1)$. This value is then introduced into eq. (13). It is clear from eq. (13) and (14) that $K_{i}^{2}(1 \rightarrow \lambda)$ is identical with $J_{i}^{2}(1 \rightarrow \lambda)$ when $E_{i}(1)$ is far from all the eigenvalues $E_{j}(2)$ and has a smoother behaviour than $J_{i}^{2}(1 \rightarrow \lambda)$ when $E_{i}(1)$ goes to one of the eigenvalues $E_{j}(2)$.

To test the validity of the two new measures of symmetry breaking, $J_{i}^{2}(1 \rightarrow \lambda)$ and $K_{i}^{2}(1 \rightarrow \lambda)$, and compare them with the old one, $I^{2}(1 \rightarrow \lambda)$, we carry out an exact shell model calculation in the framework of the exactly solvable Lipkin's model [13]. The degeneracy of both levels is taken as $N=118$. The ground state belongs to the multiplet corresponding to $J=59$ and is obtained by diagonalizing a $60 \times 60$ matrix. The 60 dimensional space includes all the states with odd values of $J_{z}$ going from - 59 up to 59 . We divide it into two subspaces $S(1)$ and $S(2)$, such that $S(1)$ includes the states with $J_{z}=-59$, - 57, - 55, - 53, - 37, - 19, - 1, 19, 31, 43, 57, and 59. This choice leads to comparatively close centroids, large internal widths and small external widths. The results $\left({ }^{1}\right)$ obtained for the total intensity of the components of $S(1)$ in the ground state in terms of the interaction parameter $N V / \varepsilon$ are displayed on figure 1. Similar results are obtained for the excited states of $\mathrm{S}(1)$ when the values of $N V / \varepsilon$ are restricted

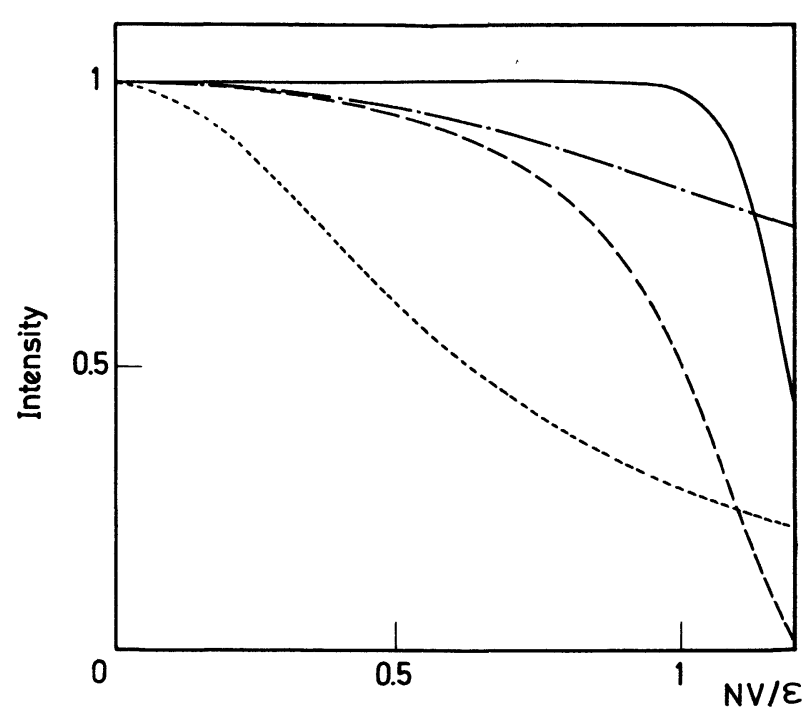

FIG. 1. - Total intensity of the components of $S(1)$ in the ground state of Lipkin's model with $N=118$ versus the interaction parameter $N V / \varepsilon$. The solid line shows the exact intensity, the dotted, short dashed, and dot-and-dash lines represent the results obtained with the $I^{2}(1 \rightarrow 2), J_{1}^{2}(1 \rightarrow 2)$, and $K_{1}^{2}(1 \rightarrow 2)$ parameters respectively.

$\left({ }^{1}\right)$ In this case as $d(1)$ and $d(2)$ are small, the matrices $\mathscr{H}_{11}$ and $H_{22}$ are exactly diagonalized. 
to those for which they are lower than the ground state of $S(2)$. The same is true also for the pairing interaction with either uncoupled or coupled quasispins.

The improvement in going from $I^{2}$ to $J_{1}^{2}$ is important for small values of the interaction parameter. However for $N V / \varepsilon$ of the order of $1, J_{1}^{2}$ becomes useless because the ground states of $S(1)$ and $S(2)$ become close to one another $\left(E_{1}(2)<E_{2}(1)\right.$ for $N V / \varepsilon \gtrsim 1.1$ ), and the first order R-S perturbation approximation is then inadequate. In this region, the replacement of $J_{1}^{2}$ by $K_{1}^{2}$, which has no effect for smaller values of $N V / \varepsilon$, becomes very useful. Up to $N V / \varepsilon \approx 1.15$, the results obtained with $K_{1}^{2}$ compare well with the exact ones. For $N V / \varepsilon \gtrsim 1.2$, the ground state of $S(2)$ is lower than that of $S(1)$ and the first order B-W perturbation approximation becomes inadequate too.

The small discrepancies which are observed between the exact results and those obtained with $K_{1}^{2}$, especially for small values of $N V / \varepsilon$, are due to the use of a sta- tistical ensemble of matrices instead of the true matrix $\mathcal{H}$. It is clear that this approximation is bad for Lipkin's interaction as well as for the pairing one, because the off-diagonal blocks $\mathscr{H}_{\lambda \lambda}, \lambda \neq \lambda^{\prime}$, contain many zeroes even when use is made of the basis which diagonalizes the diagonal blocks $\mathcal{H}_{\lambda \lambda}$.

On the other hand, it is well known that these interactions induce collective states and therefore give rise to scalar distributions which are far from gaussians [14]. The fact that $\{\mathcal{K}\}$ does not reproduce the external moments of $\mathcal{H}$ of order higher than 2 may thus be a weakness of our derivation for these interactions.

For all these reasons, we think that the agreement we get here between the exact results and those obtained with $J_{1}^{2}$ for small values of this parameter or with $K_{1}^{2}$ up to the crossing of the levels of both subspaces is quite encouraging and that it might be improved if a more realistic interaction was used.

One of us (S. S.) acknowledges the Institut Interuniversitaire des Sciences Nucléaires (Belgium) for its financial support.

\section{References}

[1] French, J. B. and Ratcliff, K. F., Phys. Rev. C 3 (1971) 94.

[2] Ratcliff, K. F., Phys. Rev. C 3 (1971) 117.

[3] Chang, F. S., French, J. B. and Thio, T. H., Ann. Phys. 66 (1971) 137.

[4] Hecht, K. T. and Draayer, J. P., Nucl. Phys. A 223 (1974) 285.

[5] Quesne, C. and SpITz, S., Ann. Phys. 85 (1974) 115.

[6] SPITZ, S., Thèse, Université Libre de Bruxelles (1976).

[7] Sitz, S. and Quesne, C., J. Physique Lett. 37 (1976) L-3; QUESNe, C. and SPITZ, S., submitted to Ann. Phys.

[8] Nissimov, H., ArVieu, R. and Bohigas, O., Nucl. Phys. A 190 (1972) 514
[9] Chang, F. S., Ph. D. Thesis, University of Rochester (1970). [10] Parikh, J. C. and Wong, S. S. M., Nucl. Phys. A 182 (1972) 593.

[11] Ullah, N., Nucl. Phys. A 164 (1971) 658.

[12] Kumar, K., Perturbation Theory and the Nuclear Many Body Problem (North-Holland, Amsterdam) 1962.

[13] Lipkin, H. J., Meshrov, N. and Glick, A. J., Nucl. Phys. 62 (1965) 188.

[14] Quesne, C., Phys. Lett. 43B (1973) 463. 